\title{
SUB-GRID PHYSICAL OPTICAL FLOW FOR REMOTE SENSING OF SANDSTORM
}

\author{
C. Cassisa $^{1,2}$, S. Simoens ${ }^{1}$, V. Prinet ${ }^{2}$, L. Shao ${ }^{1}$ \\ ${ }^{1}$ Lab. of Fluid Mechanics and Acoustics (LMFA), Ecole Centrale Lyon, France, \\ ${ }^{2}$ NLPR/LIAMA, Institute of Automation, Chinese Academy of Sciences, Beijing, China
}

\begin{abstract}
Satellite observations are currently of major importance in geosciences. Remote sensing is a strong tool to study atmospheric and earth phenomena. In this work, we propose a new motion estimation approach with application to dust storm tracking from remote sensing images. Dynamic natural phenomena in the atmosphere are generally turbulent due to a high Reynolds number. Meteorological images are still poor in time and space resolution compared to the turbulence characteristics of the flow. To tackle this problem, we define a new formulation of the flow equation based on a filtered scalar transport equation. Using Large Eddy Simulation theory, we propose a sub-grid model which incorporates small scale effects as missing (ie non-observed) information of remote sensing images. For day light changes, a uniform brightness variation term is incorporated to the model. We validated our approach on synthetic Direct Numerical Simulation (DNS) of scalar propagation. Promising results are obtained on real MTSAT-1R visible images of a dust storm event over Australia.
\end{abstract}

Index Terms - Motion estimation, Sub-grid model, Turbulence, Optical flow, Remote sensing, Duststorm

\section{INTRODUCTION}

Satellite observations are currently of major importance in geosciences. Remote sensing is a strong tool to study atmospheric and earth phenomena. In this paper, we are concerned with the analysis of dust storm events. These phenomena often appear on four of the five continents. Dust particles can be transported through the atmosphere over thousands of kilometers. Dust Storms have strong impact on health and economy of the touched regions. The estimation of the displacement of dust particle from images is important for a better understanding and modeling of this phenomenon.

Atmospheric motion from geostationary remote sensing image was well studied in meteorological community since the late 1960s [1]. Traditional methods used to estimate the displacement field are based on cross-correlation techniques [2]. These methods are easy to implement and robust to noise; however they need large interrogation windows to be able to correctly detect correct correlation peaks. Moreover they are not suitable for scalar motion estimation where correlation peaks are hardly detectable, as for exemple in clouds, dust or smoke images.

Optical Flow (OF) is a well established technique to estimate the apparant motion of objects from image sequences [6]. Because classical optical flow is not adapted to non-rigid motion, new approaches have been developed to estimate fluid motion $[3,4,5]$. They propose to define the flow equation based on the continuity equation. However, considering incompressibility property of the fluid and that dust storm displacement can be simplified has $2 \mathrm{D}$ propagation, the continuity equation can eventually be assimilated to the classical OF.
Recently, [7] incorporates Navier-Stokes equations in the regularization constraint. But this method needs long temporal acquistion at regular time intervals.

These OF approaches consider that brightness should be constant over small time interval, hence preprocessing is used to remove day light variations. In [8], the authors proposed to extend OF assumption to light variation problems using diffusion models. [9] considers brightness variation as a multiplicative and additive factor to the observed intensity.

In fact, dynamic natural phenomena in the atmosphere are generally turbulent due to a high Reynolds number. Despite relatively high image spatial resolution and temporal rate acquisition of geostationary satellites, space and time resolutions are still too low compared to turbulence characteristic dimensions of fluid motion. Classical motion estimation algorithms become weak when applied to scalar quantities transported by turbulent fluid. Only [7] inserts turbulence effects through the regularization function. But the flow equation itself does not incorporate any notion of turbulence.

In this work, we propose a new model to estimate and analyse turbulent fluid motion from remote sensing image sequences. To this end, we define the fluid flow by a scalar transport equation [10], and link the concentration of the scalar to the observed grey-level in the image. We further apply Large Eddy Simulation (LES) decomposition [11] to the scalar transport equation and model the influence of small scales with a subgrid turbulent viscosity factor. Global illumination variation from one acquisition to another is taken into account by considering the day time light changes as an additive term to the observed data.

The rest of the paper is organised as follows. We describe in section 2 our motion estimation approach. In section 3, experimental results are illustrated and validated on a Direct Numerical Simulation (DNS) sequence of scalar propagation and on a real MTSAT-1R optical image pair, which captures the 09/22/2009 dust storm event over Australia. We conclude in section 4.

\section{MOTION ESTIMATION}

In this section, we formulate the problem of motion estimation as a energy minimisation problem.

Many tasks in Computer Vision or Image Processing can be cast as an energy minimisation problem $[17,18]$. Energy-based models can capture dependencies between variables by establishing some compatibility measure between those. The energy is defined as a function of an observation variable (the image grey-level pixels in our case) and an unknown variable (the displacement vector). Both observation and unknown are defined over the image domain. The energy model itself measures the goodness (or badness) of each possible configuration of the unknown variable, knowing the observation: by convention, the best configuration is the one that minimises the energy function. 
In the present application, we aim at recovering a $2 \mathrm{D}$ velocity field $W=\{\vec{w}(s)=(u(s), v(s)) \mid \forall s \in S\}$, by minimising an equation of the form:

$$
E(W, I)=\sum_{s \in S} V_{d}(\vec{w}(s), I(s))+\alpha_{p} \sum_{s, s^{\prime} \in S} V_{p}\left(\vec{w}(s), \vec{w}\left(s^{\prime}\right)\right)
$$

where $s$ is a pixel of image domain $S$ and $s^{\prime}$ its neighbor. $V_{d}(I, \vec{w})$ is called the data term; its exact definition depends on the problem at hand (see section 2.2). $\alpha_{p}$ is a weighting coefficient that balances the influence of the term $V_{d}$ w.r.t to the prior $V_{p}$ (prior that we will define in section 2.1).

We seek for the (hopefully unique) solution $\hat{W}$ which minimises equation (1):

$$
\hat{W}=\arg \min _{W \in \mathcal{W}} E(W, I)
$$

where $\mathcal{W}$ is the entire set of possible solutions. We refer to [13] for details on the optimisation technique used to solve (2).

\subsection{Prior term}

$V_{p}$ captures the spatial dependencies between variables $\vec{w}(s)$. In equation (1), it acts as a regularisation factor. It is common to define $V_{p}$ as a "smoothness term" $[6,12]$ : it enforces spatial continuity of the velocity $\vec{w}$ by taking the norm difference of its components over a neighborhood:

$$
V_{p}\left(\vec{w}(s), \vec{w}\left(s^{\prime}\right)\right)=\left|u(s)-u\left(s^{\prime}\right)\right|^{2}+\left|v(s)-v\left(s^{\prime}\right)\right|^{2}
$$

It is a convex function of the unknown variable $\vec{w}$.

\subsection{Scalar transport equation (TE)}

The velocity field of a passive scalar is described by the scalar transport equation. In dimensionless form, it writes as follows:

$$
\frac{\partial C}{\partial t}+\nabla \cdot(C \vec{w})-\frac{1}{R e S c} \Delta C=0
$$

where $C$ is the scalar concentration field of a specie spreaded in the studied fluid. $\vec{w}=(u, v)$ is the $2 \mathrm{D}$ appearent velocity of the concentration field; space and time partial derivatives are $\nabla=\left(\frac{\partial}{\partial x}, \frac{\partial}{\partial y}\right)$ and $\frac{\partial}{\partial t} ; \Delta$ is the Laplacian operator. Re and $S c$ are the Reynolds and Schmidt numbers respectively. If we assume the fluid to be incompressible (i.e $\nabla \cdot \vec{w}=0$ ), then equation (4) becomes:

$$
\frac{\partial C}{\partial t}+\vec{w} \cdot \nabla C-\frac{1}{R e S c} \Delta C=0
$$

The concentration $C$ of a scalar quantity (dust particules, humidity, ...) transported by atmospheric wind and observed in a satellite image, can be related to the image pixel intensity value $I$. For cloud motion analysis, some authors established empirically the following relation: $I \propto \int C d z$ [14] or $I \propto\left(\int C d z\right)^{-1}$ [15], where $z$ is the observation depth.

In this paper, we consider a simpler case, and asssume that the pixel grey-level value is directly proportional to the concentration $C$ :

$$
I(s, t) \propto \alpha C(s, t)
$$

where $\alpha$ is a constant independent of time and space. In other words, we consider the $\delta z / \delta x<<1, \delta z / \delta y<<1$.

\subsection{Filtered transport equation with subgrid scale (TE-SGS)}

Images are discret projective representations of the observed 3D real world. They have limited resolution in space and time: $\delta t$ (acquisition period) and $\delta=(\delta x, \delta y)$ (pixel size) are fixed by the image acquisition conditions. Details at scales lower than the pixel are not observed: they are 'missing' information. Satellite image acquisition scales of turbulent atmospheric environment are too large compared to the characteristic scales of turbulence itself: sub-pixel displacements are important because they strongly influence the large scale motion of the observed scalar quantity. In this section, we derive a new fluid motion equation, by integrating small scales effects into the scalar transportation equation.

The approach we propose is inspired from a well-known model in fluid mechanics, namely the Large Eddy Simulation (LES) [19, 11]. One fundamental concept of LES is to postulate that the instantaneous passive scalar concentration $C$ can be decomposed into large and small scales; more precisely, it verifies: $C=C^{+}+C^{-}$ where $C^{+}$and $C^{-}$represent respectively the large scale (associated to pixel grey-level value) and the small scale (non-observed) contributions. We apply the same principle and decompose $C$ (or equivalently $I$ ) and $\vec{w}$ into small and large scales. One can further show that, after inserting this new representation into equation 4 , then only the convective term $(C \vec{w})^{+}$contains interactions of small and large scales; it writes $(C \vec{w})^{+}=C^{+} \vec{w}^{+}+\vec{\tau}$, where $\vec{\tau}$ is the residual stress tensor. $\vec{\tau}$ is embed all small scale interactions between variables.

Hence, the filtered scalar transport equation, for incompressible fluid, becomes:

$$
\frac{\partial C^{+}}{\partial t}+\vec{w}^{+} \cdot \nabla C^{+}+\nabla \cdot \vec{\tau}-\frac{1}{R e S c} \Delta C^{+}=0
$$

$\vec{\tau}$ is associated to a turbulent viscosity term [19]:

$$
\vec{\tau}=-D_{t} \nabla C^{+}
$$

where $D_{t}$ is a turbulent diffusion coefficient. Finally, inserting equation (8) into (7) leads to :

$$
\frac{\partial C^{+}}{\partial t}+\vec{w}^{+} \cdot \nabla C^{+}-\left(\frac{1}{R e S c}+D_{t}\right) \Delta C^{+}=0
$$

Such subgrid scalar transport equation (TE-SGS) formulation is a new approach for motion estimation. For high Reynolds number $(\operatorname{Re}>>1)$ or scalar with low molecular diffusion $(S c>>1)$, the contribution from turbulent diffusion becomes large compared to molecular diffusion. In our work, we define the turbulent diffusion coefficient $D_{t}$ as a statistical constant over the space domain.

\subsection{Illumination invariance}

We established, in the previous section, the relationship between the scalar concentration and the image grey-level value ( $c f$ equation 6 ): $I(s, t) \propto \alpha C^{+}(s, t)$. However, this is an over simplification : external factors such as day light variation or atmosphere humidity may lead to global changes of the observation $\bar{I}^{+}$, thought $C^{+}$may not vary.

To overcome this problem, we assume that the image's greylevel changes linearly from one acquisition to the next : $\partial I^{+} / \partial t \approx$ $\partial C^{+} / \partial t+\beta(t)$. This is equivalent to 'centerize' the temporal variation of $C^{+}$. 


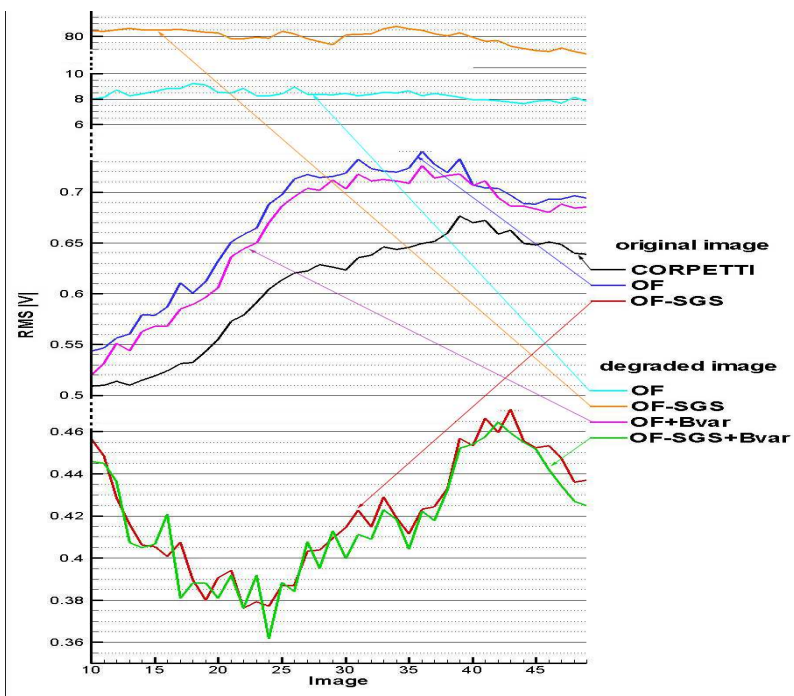

(a) RMSE for 40 successive DNS images for different methods

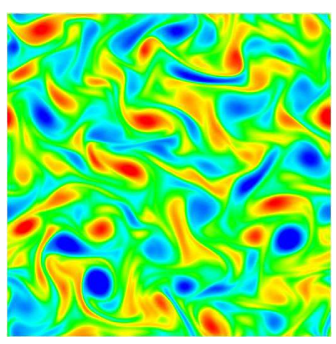

(b) Exact DNS vorticity map

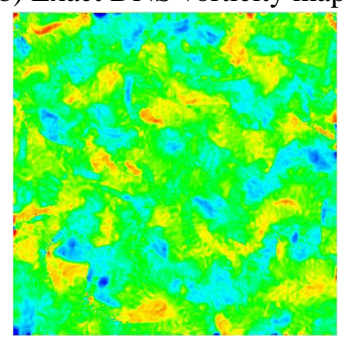

(c) [14] vorticity map (from original DNS)

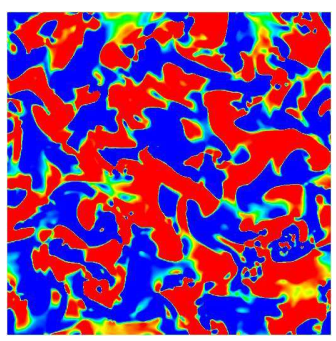

(d) $T E-S G S$ vorticity map (from degraded DNS)

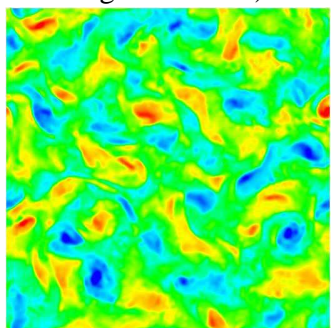

(e) $T E-S G S+B v a r$ vorticity map (from degraded DNS)

Fig. 1. Synthetic DNS results on original images for [14], TE and TE-SGS and on degraded images (as satellite kind) for $T E$ and $T E-S G S$ and for light unvariant approachs $T E+B v a r$ and TE-SGS+Bvar. Left: RMSE between estimated and exact flow field; Right: estimated vorticity map at time $t=30$ compare to exact vorticity map (b).

At last, the illumination change invariant transport equation defines our data term in (1); it writes :

$$
\begin{aligned}
& V_{d}(I(s), \vec{w}(s))= \\
& \quad\left(\frac{\partial I^{+}}{\partial t}-\beta(t)\right)+\vec{w}^{+} \cdot \nabla I^{+}-\left(\frac{1}{R e S c}+D_{t}\right) \Delta I^{+}
\end{aligned}
$$

with $\beta(t)=\frac{1}{S} \sum_{s \in S} \partial I^{+}(s) / \partial t$. We name it the + Bvar model.

\section{RESULT ILLUSTRATION}

We validate our sub-grid model (TE-SGS) on a synthetic DNS scalar sequence; we compare our estimations with an approach based on transport equation without subgrid model (namely TE, equ. 5), and with results from the state-of-the-art on fluid motion estimation [14]. In order to evaluate the efficiency of our illumination model, we simulated day light variation effect (see Figure 1) by added a spatially constant intensity offset different at each time $t$. The offset range goes from -50 to +50 on a 255 gray-level intensity image.

We computed the average Root Mean Square Error (RMSE) between the estimated velocity $\vec{w}_{e}$ and the exact velocity $\vec{w}_{c}$. RSME is computed from original and degraded images, and for each of the methods. The RMSE is defined by:

$$
R M S E=\frac{1}{N} \sum_{i}^{N} \sqrt{\left|\vec{w}_{e}-\vec{w}_{c}\right|^{2}}
$$

The DNS sequence is composed by 100 images. To suppress small scale information, only 1 every 10 time step computation is kept and input images are smoothed by a gaussian filter of variance $\sigma=1$.

On the original DNS sequence, [14] and TE give similar results because they use same kind of flow equation. In this sequence, the fluid is incompressible: div $\vec{w}=0$. Re $\approx 3000$ and $S c \approx 0.7$, then molecular diffusion is negligible compare to convective terms. In these conditions, it can be shown that the continuity equation and the transport equation are equivalent. Figure 1.b represents the exact vorticity map computed from the DSN. We observe that $T E-S G S$ (Fig.1.e) better retrieves the vorticity map than [14] (Fig. 1.c) or TE. Our model $T E-S G S$ clearly outperforms both methods. For $T E-S G S$, the turbulent diffusion coefficient is $D_{t}=0.25$.

On degraded images, where an intensity offset has been added to the sequence along the time, methods with brightness variation model + Bvar are not sensitive to the light variation. Without + Bvar model, TE give a strongly wrong estimation. TE-SGS improves the velocity estimation in turbulent scalar flows while Bvar reduces day light variation effect on the estimation.

We processed a real pair of optical images from satellite MTSAT-1R taken during the 22 sept. 2009 dust storm event that happened in Australia. Image sizes are $300 \times 300$ pixels with a resolution of $5 \mathrm{~km}$ by pixel. $\Delta t$ is 1 hour. These lenght and time scales are high compared to the atmospheric characteristic scales. On this pair of images, we compare our $T E-S G S$ estimations with results from the commercial software LaVision (which uses a crosscorrelation algorithm) [16]. Figure 2 illustrates the input pair of images, the estimated velocity field (top) and streamlines (bottom) resulting from each approach. We observe that cross-correlation technique estimates a diplacement field which is not smooth; experimentally, it needs large interrogation window size, and the correct correlation peak is difficult to localize due to slowly varying concentration. Results from our $T E-S G S+B v a r$ model give a visually smoother flow field than $T E-S G S$ which is affected by day light variation. Streamlines generated from results given by $T E-S G S+B v a r$ show the same behavior as LaVision. Dust cloud motion is more identifiable on $T E-S G S+B v a r$ results and are visually more appealing than from other methods. Advantage of our method compares to cross-correlation approaches is that the motion field is estimated from fluid kinetic equation. The estimated flow field is also dense (one velocity vector by pixel). For LaVision, final interrogation windows are $16 \times 16$ pixels. 


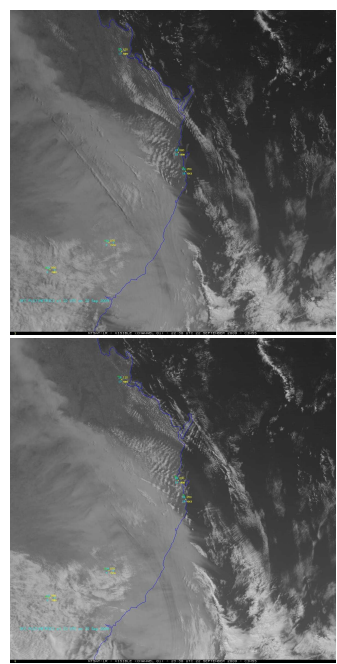

(a) 2 successive MTSAT-1R satellite images.
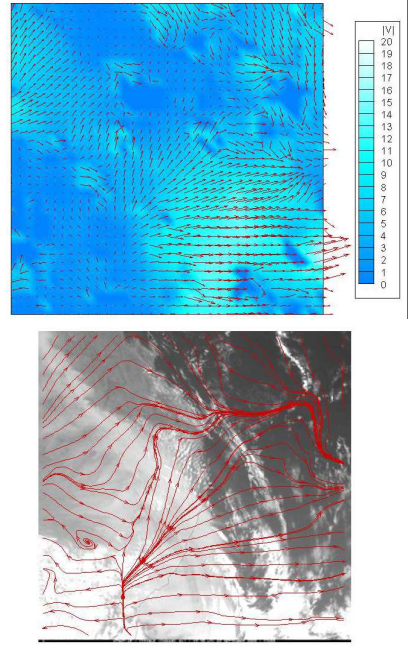

(b) LaVision cross correlation algorithm.
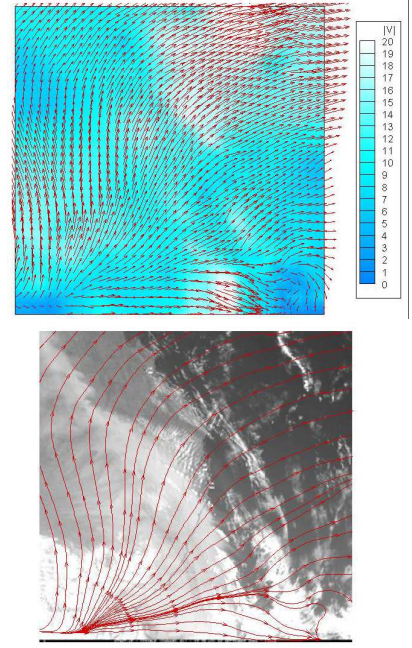

(c) $T E-S G S$

algorithm.

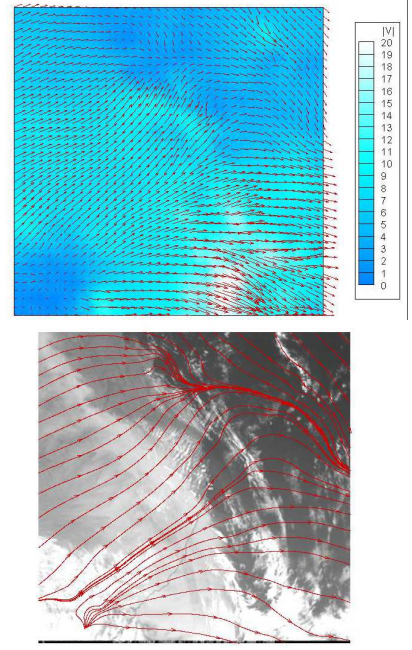

(d) $T E-S G S+B v a r$ algorithm.

Fig. 2. Australian duststorm event in 2009. Comparison of cross-correlation LaVision with TE-SGS and TE-SGS+Bvar.

\section{CONCLUSION}

In this work, we proposed a subgrid transport model to analyse atmospheric motion from remote sensing images. Different from existing methods, our model takes into account non-observed small scales effects by incorporating a turbulent diffusion term into the scalar transport equation. In satellite images, in order to compensate for the variation of global illumination from one acquisition to another, we further introduced a brightness variation correction term. Experimental results show that taking into account the turbulent component improves significantly the estimation of the displacement fields in synthetic and real images.

We are currently working on a more advanced definition of the turbulent coefficient $D_{t}$.

\section{REFERENCES}

[1] T. Fujita, "A study of mesoscale cloud motions computed from ats-1 and terrestrial photographs from satellite," Mesometeorological research project, vol. 71, pp. 25, 1968,

[2] J.A. Leese, C.S. Novack, B.B. Clark, "An automated technique for obtained cloud motion from geosynchronous satellite data using cross correlation," Journal of applied meteorology, vol. 10, pp. 118132, 1971,

[3] D. Bereziat, I. Herlin, and L. Younes, "Motion estimation using a volume conservation hypothesis," in ICASSP, 1999, pp. $3385-3388$

[4] L. Zhou, C. Kambhamettu, and D. B. Goldgof, "Extracting nonrigid motion and $3 \mathrm{~d}$ structure of hurricanes from satellite image sequences without correspondences," in ICPR, 1999, pp. 280-285.

[5] T. Corpetti, E. Menin, and Perez, "Dense estimation of fluid flow," Trans. on PAMI, pp. 365-380, 2002.

[6] B.K.P. Horn and B.G. Schunck, "Determining optical flow," Artificial Intelligence, vol. 17, no. 1-3, pp. 185-203, 1981.

[7] P. Heas, E. Memin, N. Papadakis, and A. Szantai, "Layered estimation of atmospheric mesoscale dynamics from satellite imagery," Trans. Geoscience and Remote Sensing, vol. 45, no. 12, pp. 4087-4104, 2007.

[8] H.W. Haussecker and D.J. Fleet, "Computing optical flow with physical models of brightness variation," in CVPR, 2000, pp. 760-767.

[9] Y.H. Kim, A.M. Martínez, and A.C. Kak, "Robust motion estimation under varying illumination," Image and Vision Computing, vol. 23, no. 4, pp. 365-375, 2005.

[10] L.K. Su and W.J.A. Dahm, "Scalar imaging velocimetry measurements of the velocity gradient tensor field in turbulent flows. I. Assessment of errors," Physics of Fluids, vol. 8, no. 7, pp. 1869-1882, 1996.

[11] S.A. Stanley C. Le Ribault, S. Sarkar, "Large eddy simulation of evolution of a passive scalar in plane jet," AIAA J., vol. 39, pp. 15091516, 2001.

[12] L.K. Su and W.J.A. Dahm, "Scalar imaging velocimetry measurements of the velocity gradient tensor field in turbulent flows. i. assessment of errors," Physics of Fluids, vol. 8, pp. 1869-1882, 1996.

[13] C. Cassisa, S. Simoens, and V. Prinet, "Two-frame optical flow formulation in an unwarping multiresolution scheme," in Springer-LNCS 5856 - CIARP, 2009, pp. 790-797.

[14] T. Corpetti, D. Heitz, G. Arroyo, E. Menin, and A. Santa-Cruz, "Fluid experimental flow estimation based on an optical-flow scheme," Experiments in Fluid, vol. 40, no. 1, pp. 80-97, January 2006.

[15] L. Zhou, C. Kambhamettu, and D. B. Goldgof, "Fluid structure and motion analysis from multi-spectrum $2 \mathrm{~d}$ cloud image sequences," in CVPR, 2000, pp. 744-751

[16] LaVision-DaVis, Software for Intelligent Imaging, 2005.

[17] Y. LeCun et al. "A Tutorial on Energy-Based Learning", Predicting Structured Data, MIT Press", 2006.

[18] Y. Boykov, 0. Veksler, R. Zabih, "Approximate Energy Minimization via Graph Cuts", IEEE Trans. PAMI, vol. 23, p.12221239, 2001.

[19] P. Sagaut, "Large Eddy Simulation for Incompressible Flows", 3rd ed., Springer, Berlin, 2006. 